This item was submitted to Loughborough's Research Repository by the author.

Items in Figshare are protected by copyright, with all rights reserved, unless otherwise indicated.

\title{
A hybrid approach to determining cornea mechanical properties in vivo using a combination of nano-indentation and inverse finite element analysis
}

PLEASE CITE THE PUBLISHED VERSION

http://dx.doi.org/10.1016/j.jmbbm.2013.05.016

PUBLISHER

(C) Elsevier

VERSION

AM (Accepted Manuscript)

\section{LICENCE}

CC BY-NC-ND 4.0

\section{REPOSITORY RECORD}

Abyaneh, M.H., Ricky D. Wildman, lan A. Ashcroft, and Pablo D. Ruiz. 2019. "A Hybrid Approach to Determining Cornea Mechanical Properties in Vivo Using a Combination of Nano-indentation and Inverse Finite Element Analysis". figshare. https://hdl.handle.net/2134/14035. 
This item was submitted to Loughborough's Institutional Repository (https://dspace.lboro.ac.uk/) by the author and is made available under the following Creative Commons Licence conditions.

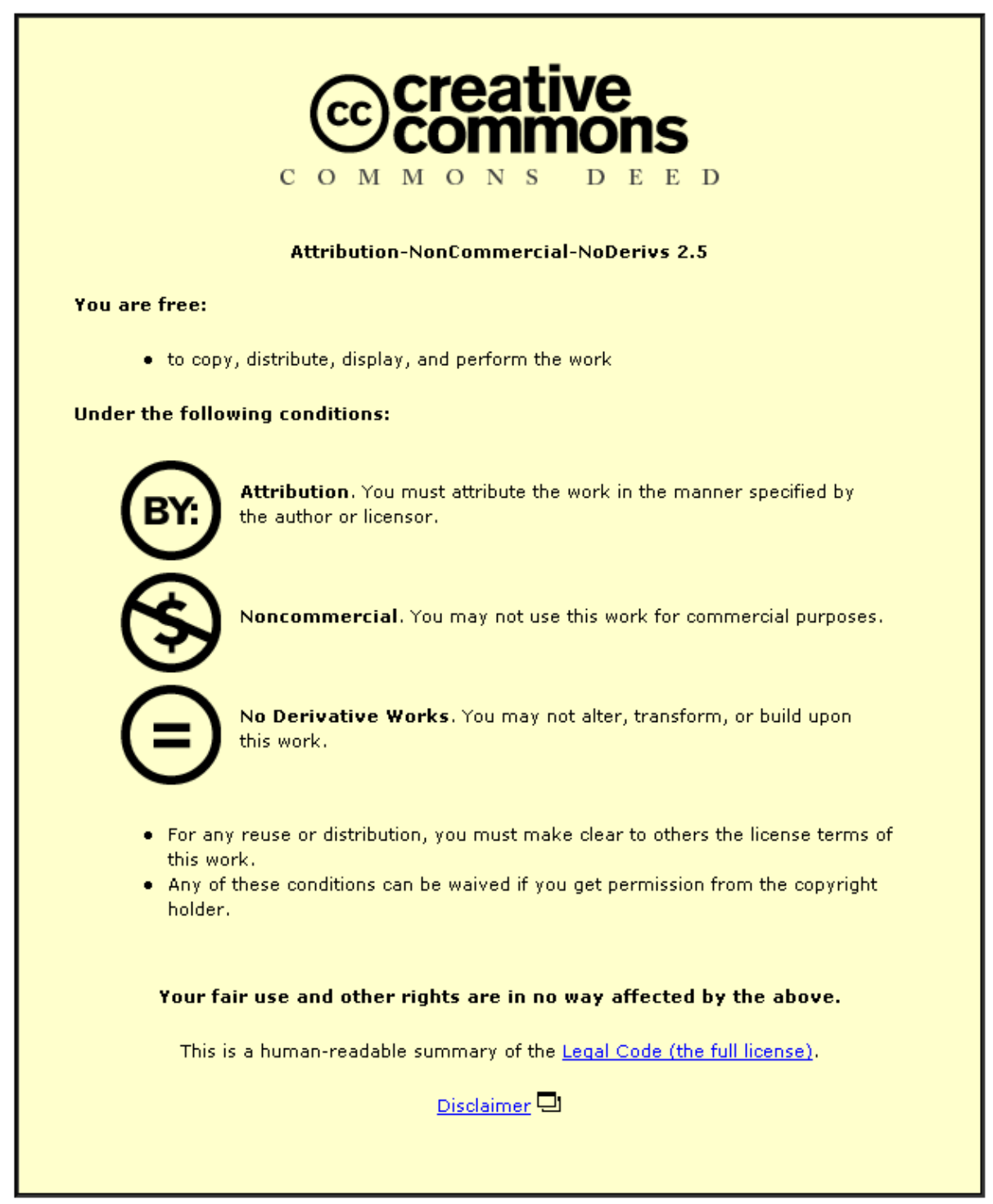

For the full text of this licence, please go to: http://creativecommons.org/licenses/by-nc-nd/2.5/ 
A hybrid approach to determining cornea mechanical properties in vivo using a combination of nano-indentation and inverse finite element analysis

Affiliation

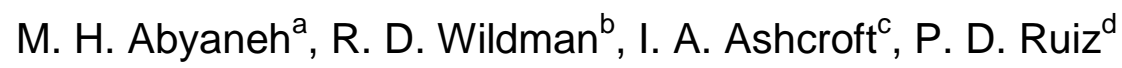

${ }^{a}$ Wolfson School of Mechanical and Manufacturing Engineering, Loughborough University, Loughborough, LE11 3TU, UK

${ }^{b}$ Faculty of Engineering, University of Nottingham, Nottingham, NG7 2RD, UK Corresponding Author:

M. H. Abyaneh

Wolfson School of Mechanical and Manufacturing Engineering, Loughborough University, Loughborough, LE11 3TU, UK

M.Haghihi-Abayneh@lboro.ac.uk

Tel: 00441509227534 
Journal of the Mechanical Behavior of Biomedical Materials

Abstract

An analysis of the material properties of porcine corneas has been performed. A simple stress relaxation test was performed to determine the viscoelastic properties and a rheological model was built based on the Generalized Maxwell (GM) approach. A validation experiment using nano-indentation showed that an isotropic GM model was insufficient for describing the corneal material behaviour when exposed to a complex stress state. A new technique was proposed for determining the properties, using a combination of nano-indentation experiment, an isotropic and orthotropic GM model and inverse finite element method. The good agreement using this method suggests that this is a promising technique for measuring material properties in vivo and further work should focus on the reliability of the approach in practice.

"Keywords

Porcine cornea; Nano indentation; Stress relaxation; Inverse finite element method 
Graphical abstract

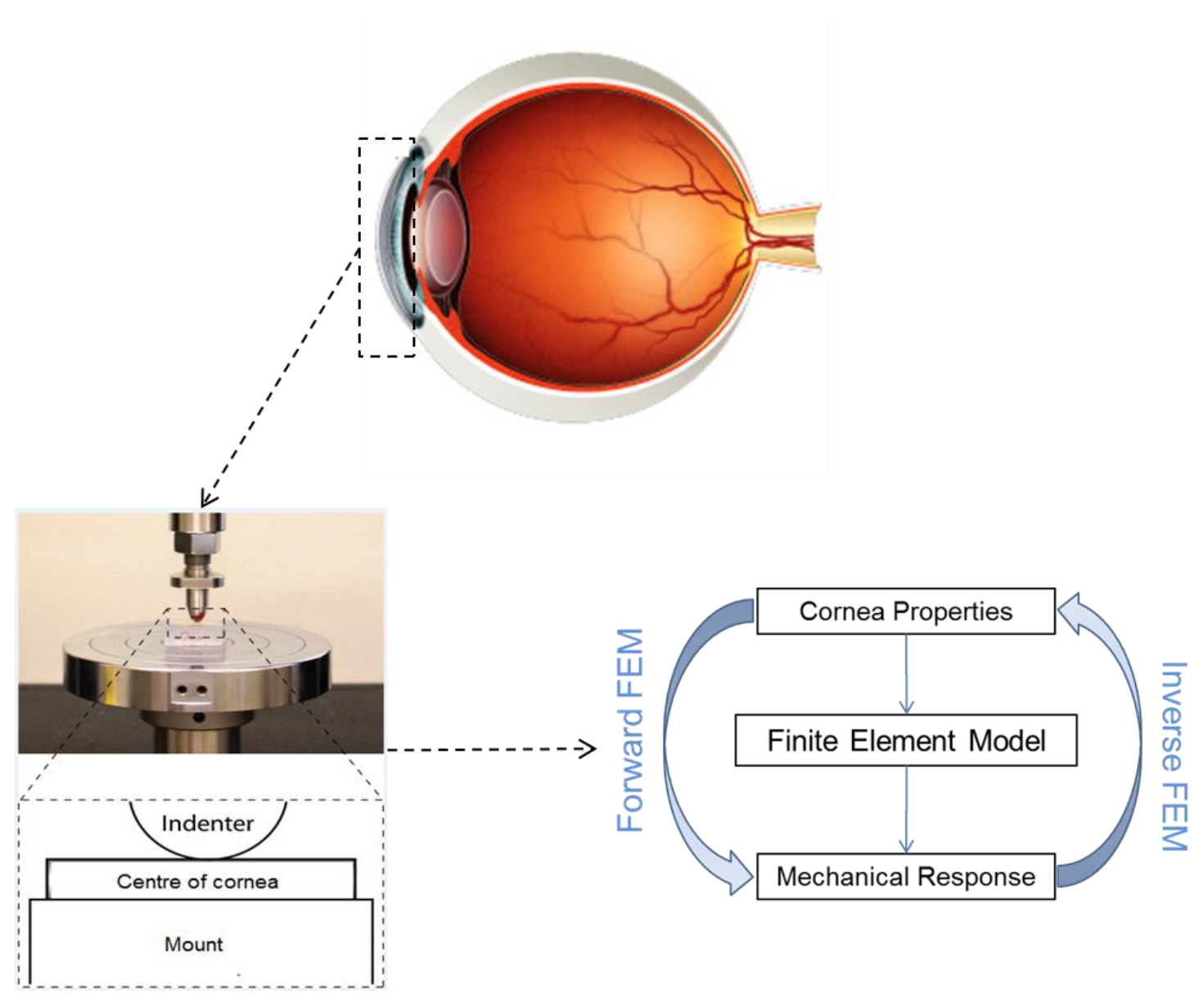

\section{Highlights}

1- A new technique was proposed for determining the properties, using a combination of nano-indentation, an isotropic and orthotropic GM model and inverse finite element method

2- This approach enables determination of all the viscoelastic parameters for a cornea

3- To investigate the performance of the inverse method, the recovered parameters were checked against the fitted parameters from the stress relaxation experiment. 
Journal of the Mechanical Behavior of Biomedical Materials

\section{Abbreviations}

GM

Generalized Maxwell

Inverse-FEM

Inverse Finite Element Method

$\mathrm{NI}$

Nano-Indentation

NT

Nasal-Temporal

Relax.

Relaxation

SIA

Surgically Induced Astigmatism

UCVA

Uncorrected Visual Acuity 


\section{Introduction}

Refractive eye surgery is used to reduce common vision disorders by changing the curvature of the corneal anterior surface. However, the achieved outcome can differ from the planned outcome due to the mechanical deformation of the cornea. For example in modern cataract surgery techniques, surgically induced astigmatism (SIA) remains a common obstacle to achieve excellent uncorrected visual acuity (UCVA). Various factors such as wound size, location, and architecture are known to affect postoperative astigmatism (Hayashi, Yoshida and Hayashi 2009). The corneal incision causes local load redistribution and consequently changes the curvature of the cornea (Pinsky et al. 2005). It is possible, however, to avoid such negative consequences if the mechanical response of the cornea could be modelled in such a way as to indicate the likely outcome of a procedure. This is possible in principle, when the constitutive models of the cornea are available and the material properties can be measured in vivo for individuals.

In general, to obtain the material properties and derive a constitutive model for biological tissues such as the cornea, two main approaches have been attempted. A classical approach is to conduct standard experiments such as tensile and compression tests, which involve in vitro measurements with significant simplifications and assumptions (Boyce et al. 2007, Hoeltzel et al. 1992, Kampmeier et al. 2000, Zeng et al. 2001). For example, to characterize biological materials with a tensile test, the material is often simplified down to homogeneity (Boyce et al. 2007). A more recent approach is to combine numerical and experimental techniques to compute the material properties indirectly such as using an inverse finite element updating method (Friswell and Mottershead 1995) or the more recently developed virtual fields method (Grédiac et al. 2006). In the inverse finite element 
updating method, which is the scope of this research, a relevant mechanical response is an input to an optimization algorithm and the parameters of the rheological model are recovered iteratively. This technique is potentially noninvasive. It can, in principle, be implemented in situ and can account for the assumptions made in standard experiments, such as homogeneity. A potential drawback of this method is that it may still require in vitro measurement of the material properties in order to inform the correct choice of rheological model, but once this has been validated, no further in vitro testing is required.

A number of studies have used standard experiments such as tensile and inflation tests to characterize the cornea. They have shown that the cornea has nonlinear viscoelastic behaviour over a large range of pressures (Boyce et al. 2007, 2008, Glass et al. 2008). Nguyen et al. have developed a complete nonlinear viscoelastic rheological model for the cornea that considers its anisotropy (Nguyen et al. 2008). However, corneal rheology can be approximated to linear viscoelasticity within the physiological range of pressure (2-4kPa) (Boyce et al. 2008, Kaliske 2000). Despite strong evidence for corneal viscoelastic behaviour and the available analytical models, few researchers have investigated its time dependent behaviour, as most consider the behaviour to be elastic or hyperelastic, which implicitly assumes the cornea has time-independent behaviour (Boyce et al. 2008, Elsheikh et al. 2007, Nguyen and Boyce 2011). One reason for this limitation is that the inflation test is generally incapable of being used to acquire corneal time-dependant response, and thus, the viscoelastic behaviour of the cornea cannot be studied.

One method to overcome the drawbacks of the inflation test is to utilize micro or nano-indentation techniques, which are similar to Goldman applanation tonometry in the way they introduce surface-to-surface contact or punch (Zadok et al. 1999). In 
order to characterize a material's time-dependent behaviour based on indentation data, typically one assumes a viscoelastic rheological model and uses that to modify the elastic Herzian contact model (Oyen 2006, Cheng et al. 2005, Yang et al. 2004). The main drawback for this method exists within the choice of the creep function (Yang et al. 2004). The creep function is typically derived for uniaxial mode of experiment (tensile or compression) under the condition of constant engineering stress, whereas in an indentation test, the material is subjected to rather a more complex mode of stress, hence, the creep function developed for uniaxial tests is not suitable to define the indentation creep. Other researchers, such as Ahearne et al. have studied the viscoelastic properties of the cornea by performing the micro indentation test based on Fung's quasi-linear viscoelastic analytical model (Ahearne et al. 2007) (Fung 1993).

An alternative is to avoid the assumptions regarding the stress state and the homogeneity and use the inverse finite element updating method. This is predicated on having the correct constitutive model, but with this in place, it is a powerful method for characterizing the time-dependent behaviour of biological materials. In this study, we propose a new method that obtains the properties of the cornea based on a combination of nano-indentation technique and inverse finite element analysis. It will be shown that the stress relaxation experiment is insufficient on its own to fully characterize corneal properties requiring the use of inverse finite element analysis coupled with an orthotropic constitutive model. The paper concludes with a demonstration that this approach enables determination of all the viscoelastic parameters for a cornea in a single indentation step. 


\section{Experimental Methodology}

In order to obtain the material properties of corneas, two experimental approaches were explored. First, several bulk tests were performed using stress relaxation experiment, to obtain the optimal experimental conditions (strain rate). Having found the optimum strain rate, one test was performed on the left corneal strip of the pair in order to obtain macroscopic properties. Secondly, nano-indentation was performed to induce a complex stress state within the cornea and was utilised to validate the proposed model and to serve as the basis for in vivo tests.

\subsection{Sample Preparation}

The testing required careful preparation of samples to ensure reliability and stability, particularly in the case of the nano-indentation tests. The samples were prepared from a pair of porcine eyeballs that were obtained from a local butcher within 24 hours of slaughter. Any experiments were then performed within 24 hours of receipt. During this time, the cornea was stored in cotton cloth sheets saturated with saline solution (Bausch \& Lomb Eyes Saline Solution, Tetronic@1304) at 5². Prior to the experiments, the corneal epithelium layer was removed, because this layer was damaged after the slaughter of the animal. This was also reported in the literature (Boyce et al. 2007).

The sample for the stress relaxation test was obtained from one rectangular nasaltemporal strip cut from the left cornea. The nasal-temporal strip provided a better grip space than the inferior-superior strip for the tensile machine, as the porcine cornea has a longer diameter along the nasal-temporal meridian. After the corneal strip was gripped at the scleral region (up to the border of the cornea), the dimensions were measured at different points along the length with a digital calliper. 
Thickness measurement were taken at 5 points and the mean was found to be $1.40 \mathrm{~mm}+/-0.13 \mathrm{~mm}$. The width was also measured at the same points with the mean value of $2.30 \mathrm{~mm}$ and STD (Standard Deviation) of $0.07 \mathrm{~mm}$. The length was measured once to be $13 \mathrm{~mm}$.

For the nano-indentation test, one whole cornea (right cornea of the pair) with a small portion of sclera was extracted from the right eyeball. The cornea was then mounted on a bulge of blue tack and glued around the scleral region (Figure 1b) to help maintain its original curvature and provide a support. Figure 1 shows the corneal samples for the stress relaxation and the nano-indentation tests.

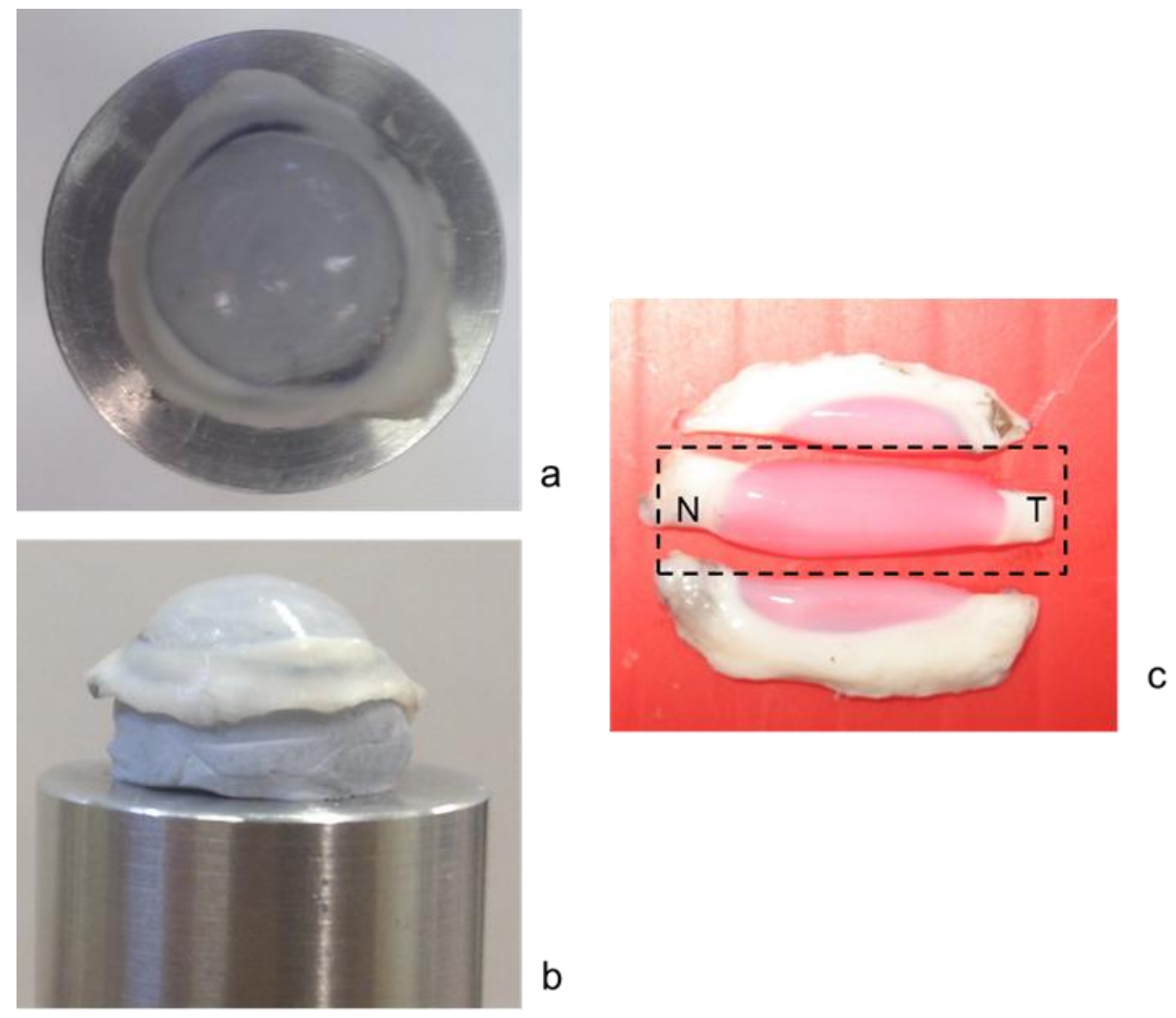

Figure 1 Corneal samples for the nano-indentation and the stress relaxation tests, a) top view of the right cornea mounted on bulge of blue tack used for the nanoindentation test, b) side view of the right cornea mounted on bulge of blue tack, c) the nasal-temporal (NT) strip of the left cornea used for the stress relaxation test 


\subsection{Experimental Procedure}

A tensile stress relaxation test was performed using an Instron 4858 Micro-Tester machine. To maintain the moisture of the sample during a test, the cornea was sprayed with the saline solution regularly. The corneal strip was pulled to $5 \%$ strain with a range of true strain rates and then held at a constant strain over 2000s, allowing the stress to relax (see schematic shown in Figure 2a).

The nano-indentation test was performed with a Nano-Tester 600 machine (Micro Materials, Wrexham UK) using a $600 \mu \mathrm{m}$ radius spherical steel indenter, at room temperature and $70 \% \mathrm{RH}$. The cornea was indented three times at points with $50 \mu \mathrm{m}$ horizontal distance apart from each other. The indentation points were adjusted via a microscope to be at the centre of the cornea and perpendicular to the indenter.

During the experiment, a thin layer of moisture was observed on the surface of the cornea after moisture equilibrium was reached, a feature important for the description of contact mechanics (Section 3.1).

A trapezoidal loading scheme (loading, holding and unloading) was employed with a depth control method (Figure $2 \mathrm{~b}$ and $2 \mathrm{c}$ ). The load was applied at the rate of $0.05 \mathrm{mN} / \mathrm{s}$ until a $5000 \mathrm{~nm}$ depth was reached then, kept constant for $200 \mathrm{~s}$, followed by $80 \%$ removal of the maximum load at the same rate. 


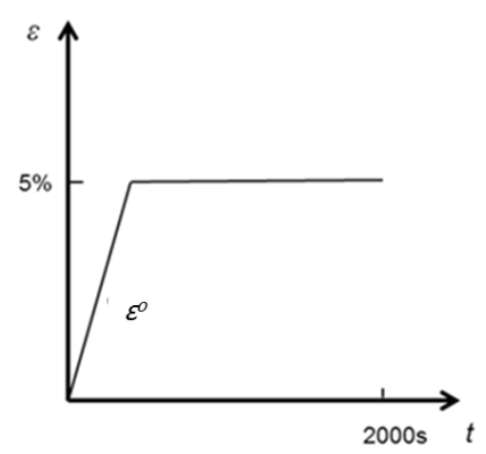

a

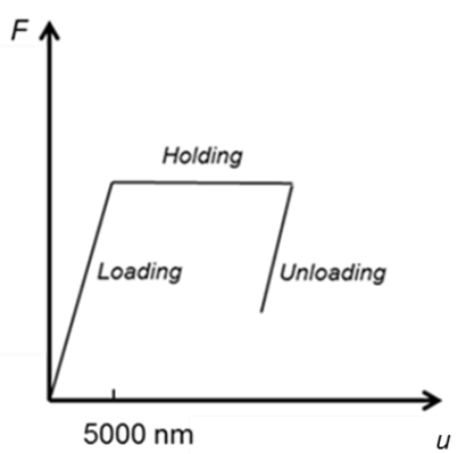

b

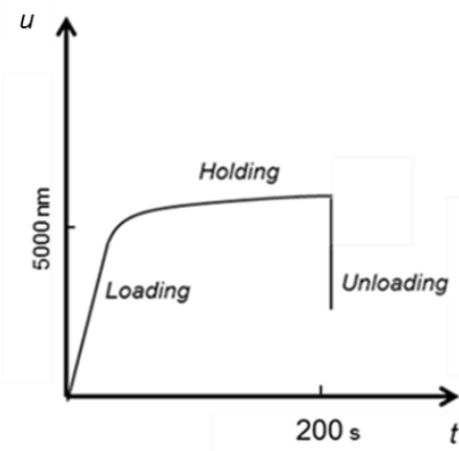

C

Figure 2 Schematic of stress relaxation and nano-indentation loading schemes, a)

Strain, $\varepsilon$, vs. time, $t$, for relaxation b) Load, $F$, vs. depth, $u$, for indentation, c) Depth, $u$, vs. time, $t$, for indentation

An important issue in the nano-indentation test was to identify the corneal contact surface prior to the experiment as part of the calibration procedure. This is important for the machine's accurate measurement of the indentation depth. To identify the contact surface, the sample is moved toward the indenter with a speed of $0.1 \mu \mathrm{m} / \mathrm{s}$. When the force registers in excess of $0.01 \mathrm{mN}$, it was assumed that the indenter was in contact with the material. This was found based on several error and trial attempts and was consistent and repeatable. Figure 3 shows the set-ups of the stress relaxation and the nano-indentation experiments. 

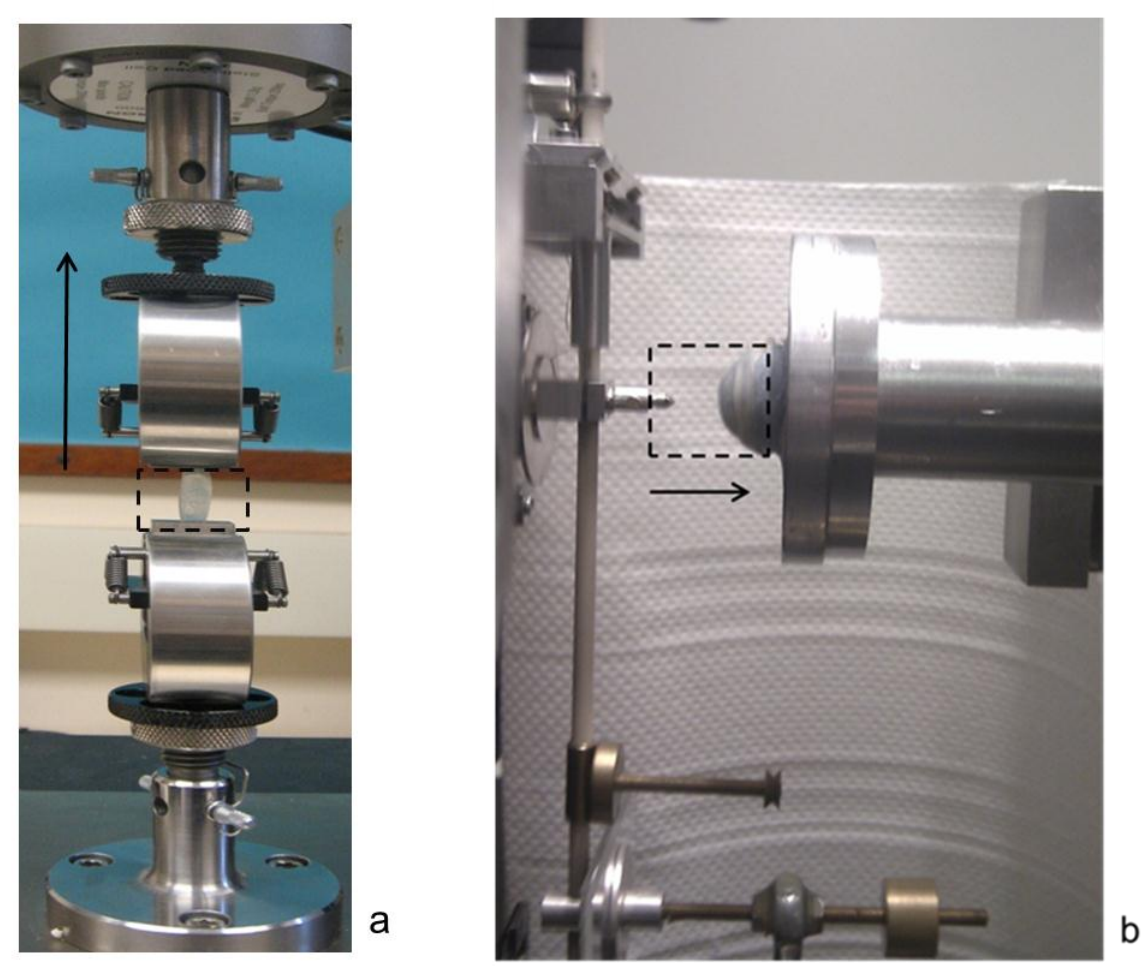

Figure 3 Experimental configurations for the stress relaxation and the nano-

indentation experiments. The arrows indicate the direction of loading and the bound area highlight the position of the sample. a) Tensile stress relaxation test, b) Nanoindentation test, showing the indenter on the left and the sample on the right of the box, which are in position prior to surface detection.

\section{Modelling}

\subsection{Isotropic Viscoelastic Model}

As a first step to understanding the cornea mechanics, a corneal linear viscoelastic model was formulated in the form of Generalized Maxwell (GM) model (Nguyen et al. 2008), which describes the stress-strain relationship by analogy with a series of 
springs and dashpots (Maxwell elements) aligned parallel to a single spring (Figure 4).

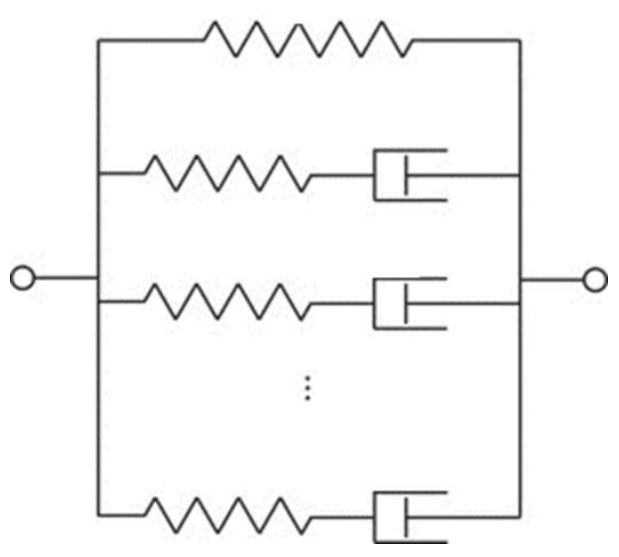

Figure 4 Schematic of the Generalized Maxwell model for linear viscoelastic material

One advantage of the GM model is that it is able to describe the stress relaxation behaviour of a material by separating the instantaneous elastic behaviour from the long-term viscoelastic behaviour. A useful starting point for many viscoelastic materials is to define the behaviour in terms of a Prony series that can capture multiple time scales of response (Liu et al. 2011). We defined the instantaneous elastic behaviour by means of a simple Hookean linear elastic model and the longterm viscoelastic part of the deformation by a Prony series expansion of shear relaxation moduli such that,

$G_{R}(t)=G_{0}-\sum_{i=1}^{N} G_{i}\left(1-\exp \left(-\frac{t}{\tau_{i}}\right)\right)$

where $G_{R}(t)$ is the shear relaxation modulus with respect to time, $G_{0}$ is the instantaneous elastic shear relaxation modulus. To make equation 1 independent 
from the elastic component, it can be written in dimensionless form by normalizing with respect to $G_{0}$,

$g_{R}(\mathrm{t})=1-\sum_{i=1}^{N} g_{i}\left(1-\exp \left(-\frac{t}{\tau_{i}}\right)\right)$

The terms $g_{i}$ and $\tau_{i}$ are the material constants. $g_{i}$ is the normalized shear relaxation modulus at the time $t$ and $\tau_{i}$ is the relaxation time corresponding to the relevant $g_{i}$.

Equation 2 is known as Prony series expansion of the normalized shear relaxation modulus. In order to study the stress relaxation behaviour of the material, we performed uniaxial tensile tests, rather than shear tests. Based on von Mises criterion of principal stresses at small deformations, the shear relaxation modulus for a material under tension can be obtained by,

$G_{R}(t)=\frac{E_{R}(t)}{2(1+v)}$

where $v$ is Poisson's ratio and $E_{R}(t)$ is elastic relaxation modulus in respect to time that is obtained via

$E(t)=\frac{\sigma(t)}{\varepsilon}$ Eq. 4

where $\sigma(t)$ is the time dependent normal stress and $\varepsilon$ is the normal strain.

An advantage of this approach means that we can separate the linear viscoelastic parameters for the cornea and can attribute them individually to different microstructural components, i.e. fibres and matrix. Parameter $E$ can be used to describe the combined response of the corneal fibres and matrix (Dunn and Silver 1983, Edmund 1988), whereas when appropriately transformed, $g_{i}$ and $\tau_{i}$ can 
describe corneal matrix behaviour (Dunn and Silver 1983, Crabb et al. 2006, Zhou et al. 2011).

The above viscoelastic description was used in an isotropic finite element model of the nano-indentation experiment. Initially, a comparative numerical study was performed in order to determine a corneal domain and ascertain the degree of influence of the blue tack on the simulated results. Two axisymmetric models were produced using Abaqus 6.11, one with full corneal thickness and a supporting base layer of blue tack and one with partial thickness. The result obtained from the two models show a negligible effect of the blue tack base and the full thickness.

Therefore, the partial domain of $1.2 \times 0.8 \mathrm{~mm}^{2}$ with 4500 , quadratic quadrilateral elements was used for further analysis (Figure 5). The corneal domain was assumed flat as the indentation area was very small in comparison to the total corneal curvature. The indenter was modelled as a rigid body with a radius of $0.6 \mathrm{~mm}$. The cornea was fixed along the axis of symmetry, $y$, for $x$ translation and along the base line for $x$ and $y$ rotation and translation. Adaptive meshing was implemented for the region of the cornea under the largest deformation. The contact was assumed frictionless based on the observation of a thin layer of water on the surface of the cornea. 


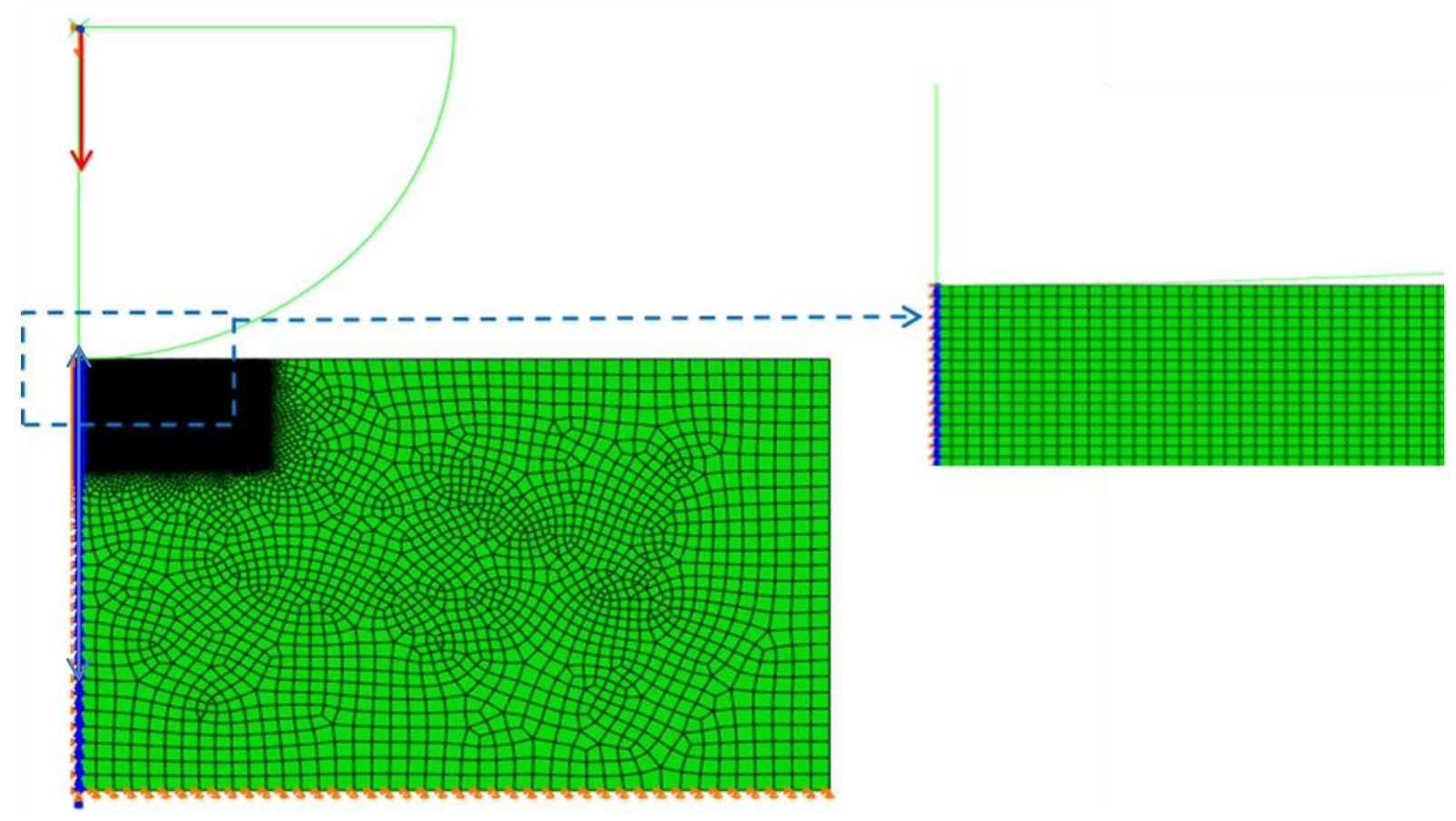

Figure 5 Finite element domain of the cornea in nano-indentation, showing magnification of the refined mesh area

\subsection{Orthotropic Finite Element Model}

To extend the isotropic model, a 3D orthotropic model was created to account for the composite microstructure of the cornea and to allow for fibre orientations. Since the instantaneous elastic behaviour of the cornea could be separated from the viscoelastic part, which could be obtained from the procedure in Section 3.1, the orthotropic model was restricted to the elastic part only. A corneal domain of $1.2 \times$ $0.8 \times 0.8 \mathrm{~mm}^{3}$ was meshed with 300008 -node quadratic brick elements using the mesh refinement region for the area under indentation. The boundary conditions and the contact specifications were defined in the same way as for the isotropic model (Section 3.1). 
The central region of cornea around the $y$-axis has an orthotropic fibre orientation with fibres aligned orthogonally in the $x z$ plane (Figure 6). It is assumed that the properties in the orthogonal directions are equal.

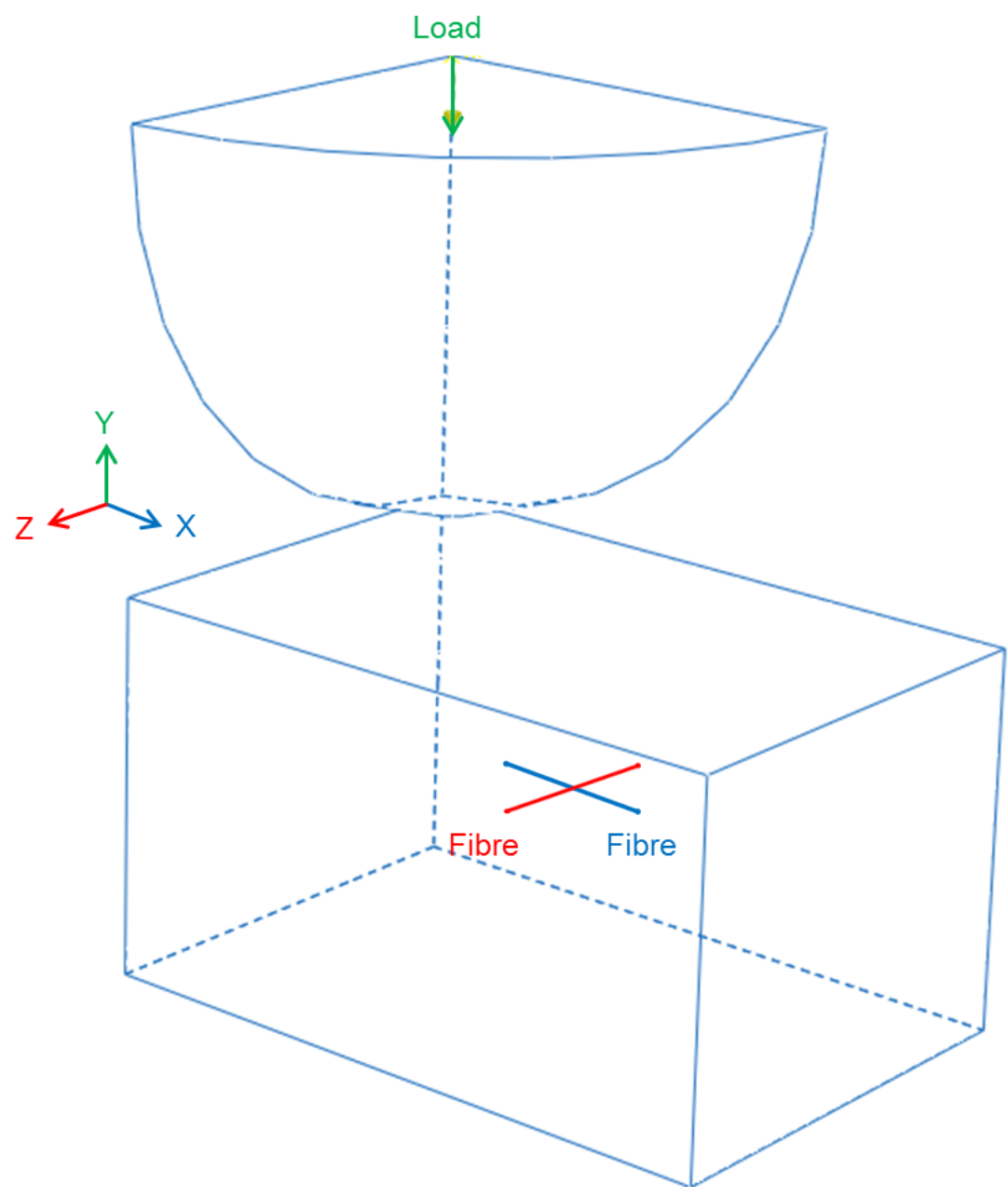

Figure 6 Orthotropic model of the central cornea in nano-indentation. Fibres are aligned along the $x$ and $z$ axes

The elastic behaviour of the cornea can be written in the form of Hooke's law as,

$$
\boldsymbol{\varepsilon}=\mathbf{S} \boldsymbol{\sigma}
$$

where $\boldsymbol{\varepsilon}, \boldsymbol{\sigma}$ and $\boldsymbol{S}$ are strain, stress and compliance tensors. An orthotropic, Hookean solid is characterised by 9 constants, $E_{x}, E_{y}, E_{z}, G_{x y}, G_{y z}, G_{x z}, v_{x y}, v_{y z}$ and $v_{x z}$. For 
the orthotropic cornea, $E_{x}$ and $E_{z}$ are the moduli along the fibres directions, $x$ and $z$. $E_{y}$ is the modulus along the loading direction and can be considered normal to the direction of fibres embedded in the matrix (Figure 6). Parameter $E_{y}$ is deduced from the isotropic instantaneous elastic constant, i.e. $E_{y}=E_{\text {isotropic }}$. The following symmetric condition is then assumed:

$E_{x}=E_{z}$

$G_{x y}=G_{z y}$

$v_{x y}=v_{z y}$

where $E_{i}, G_{i j}$ and $v_{i j}$ are elastic modulus, shear modulus and Poisson's ratio corresponding to the planes of symmetry, i.e. $x y$ and zy planes. Further, using an assumption of corneal incompressibility i.e., $v_{x y}=v_{z y}=0.5$ (Nguyen et al. 2008), Poisson's ratio and shear modulus on the plane of orthotropy, $v_{x z}$ and $G_{x z}$ can be obtained from (Itskov and Aksel 2002),

$v_{x z}=1-v_{x y}$ Eq. 6

$G_{x z}=\frac{E_{x}}{2\left(1+v_{x z}\right)}$

Thus, the orthotropic elastic cornea is characterized by four independent parameters, $E_{x}, E_{z}, E_{y}$ and $G_{x y}$. Parameter $G_{x y}$ was recently measured by Nickerson (Nickerson 2006), who suggested a value of $G_{x y}=3 \mathrm{~Pa}$. Parameter $E_{y}$ will be obtained from the isotropic model (explained in section 4) leaving only $E_{x}$ and $E_{z}$ to be obtained from the orthotropic model. 


\subsection{Inverse Method}

An inverse finite element updating method was used to first obtain the parameters $E=E_{y}, g_{1}, g_{2}, \tau_{1}$ and $\tau_{2}$ from the isotropic model and then to find the instantaneous elastic parameters in the orthogonal directions along fibres, i.e. $E_{x}$ and $E_{z}$, from the orthotropic model. In general, the inverse method is a mixed numerical-experimental technique to recover material properties iteratively by minimising a relevant objective function. In this inverse analysis, the objective is to minimize the root mean square (rms) difference between the experimental indentation depth and the numerical nodal displacement at successive time steps. The objective function is, hence,

$$
\operatorname{Min}: \Delta=\sqrt{\frac{1}{N} \sum_{i=1}^{N}\left(U(t)_{F E A i}-U(t)_{E x p i}\right)^{2}}
$$

Abaqus explicit was used as the numerical solver, called through a Matlab® algorithm. The algorithm uses a gradient-based variable updating scheme (Levenberg 1944). Tolerances for the objective function and the variable change were less than $10^{-8}$ and $10^{-6}$, respectively. A flowchart for the inverse method developed in this study is provided in Figure 7. 


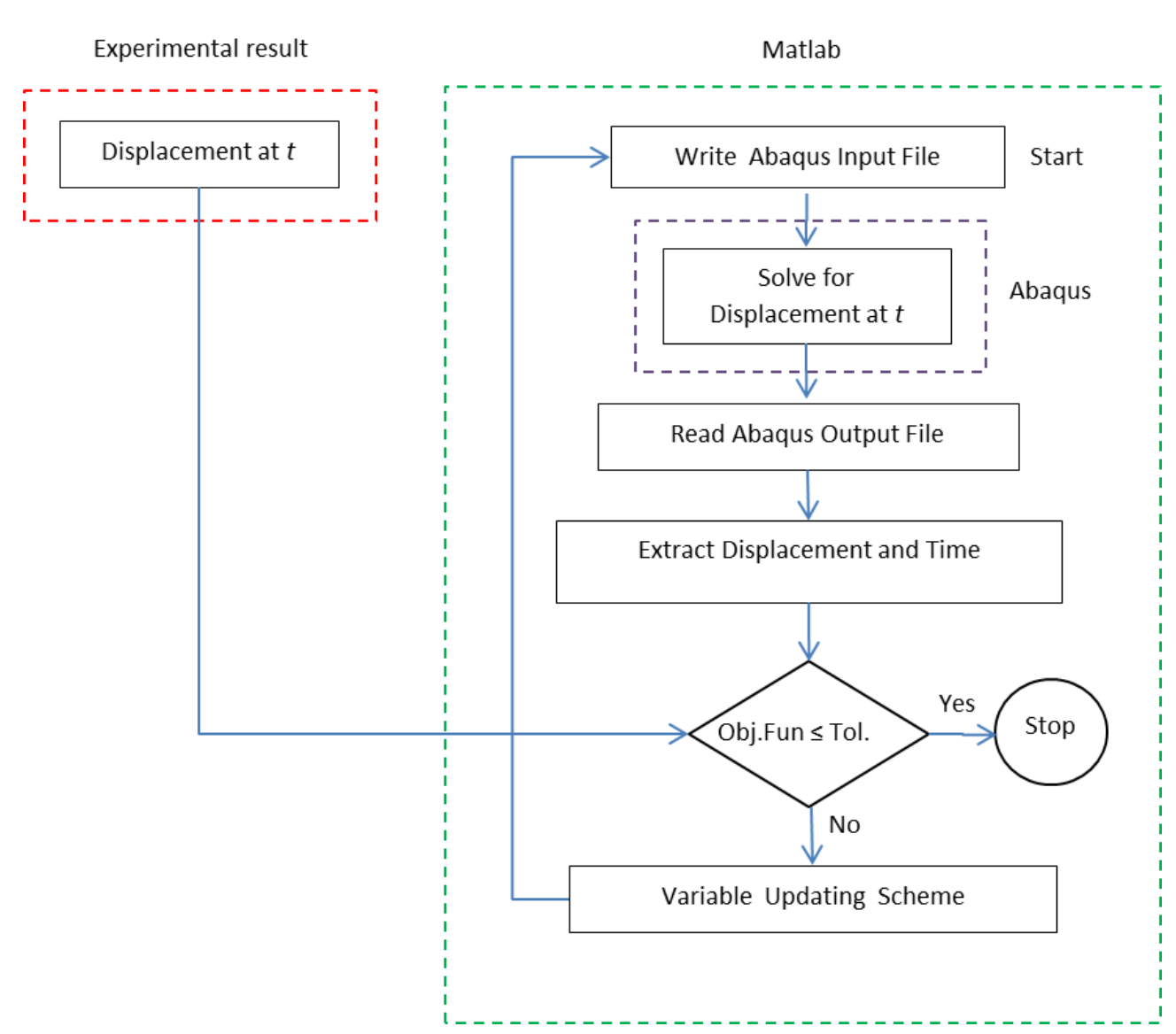

Figure 7 Flowchart of the inverse method used to determine corneal material properties

\section{Results and Discussion}

The viscoelastic properties were obtained from a stress relaxation test performed on the left porcine cornea. A stress relaxation curve consists of two parts (shown separately): an initial pulling part (Figure 8) and a decay part (Figure 9). Initially, a range of strain rates were employed in order to find the minimum strain rate required for the material to perform in an approximately linear-elastic manner. This was found to be $0.1 / \mathrm{s}$. Later, one test was performed (on the left corneal sample) at this strain rate to obtain the curves required for the model fitting. Figure 8 shows the pulling 
part of the stress vs. strain curves at different strain rates.

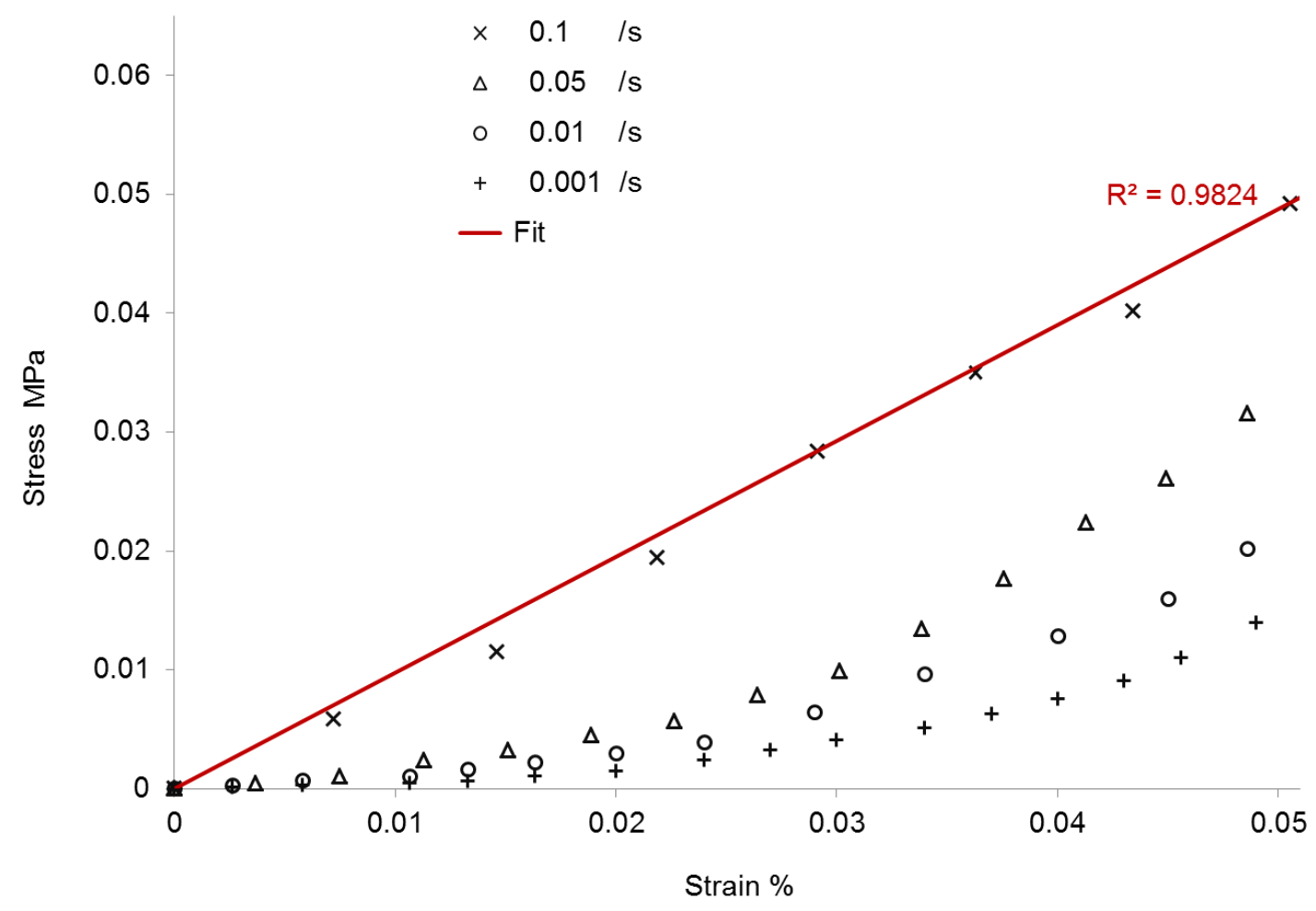

Figure 8 Stress relaxation results of the porcine cornea at various strain rates showing a linear response at a strain rate of $0.1 / \mathrm{s}$. The linear elastic model is fit to the data at the strain rate of 0.1 /s to find the instantaneous elastic parameter

The instantaneous elastic modulus, $E$, was inferred from the initial part of the stressstrain curve at $0.1 / \mathrm{s}$, where a linear line with correlation coefficient of $R^{2}=0.9824$, was fitted to the data (Figure 8). Two terms in the Prony series were employed and this was fitted to the normalized time-dependent shear moduli curve (decay part), as shown in Figure 9. In total, five parameters were obtained which are presented in Table 1. 


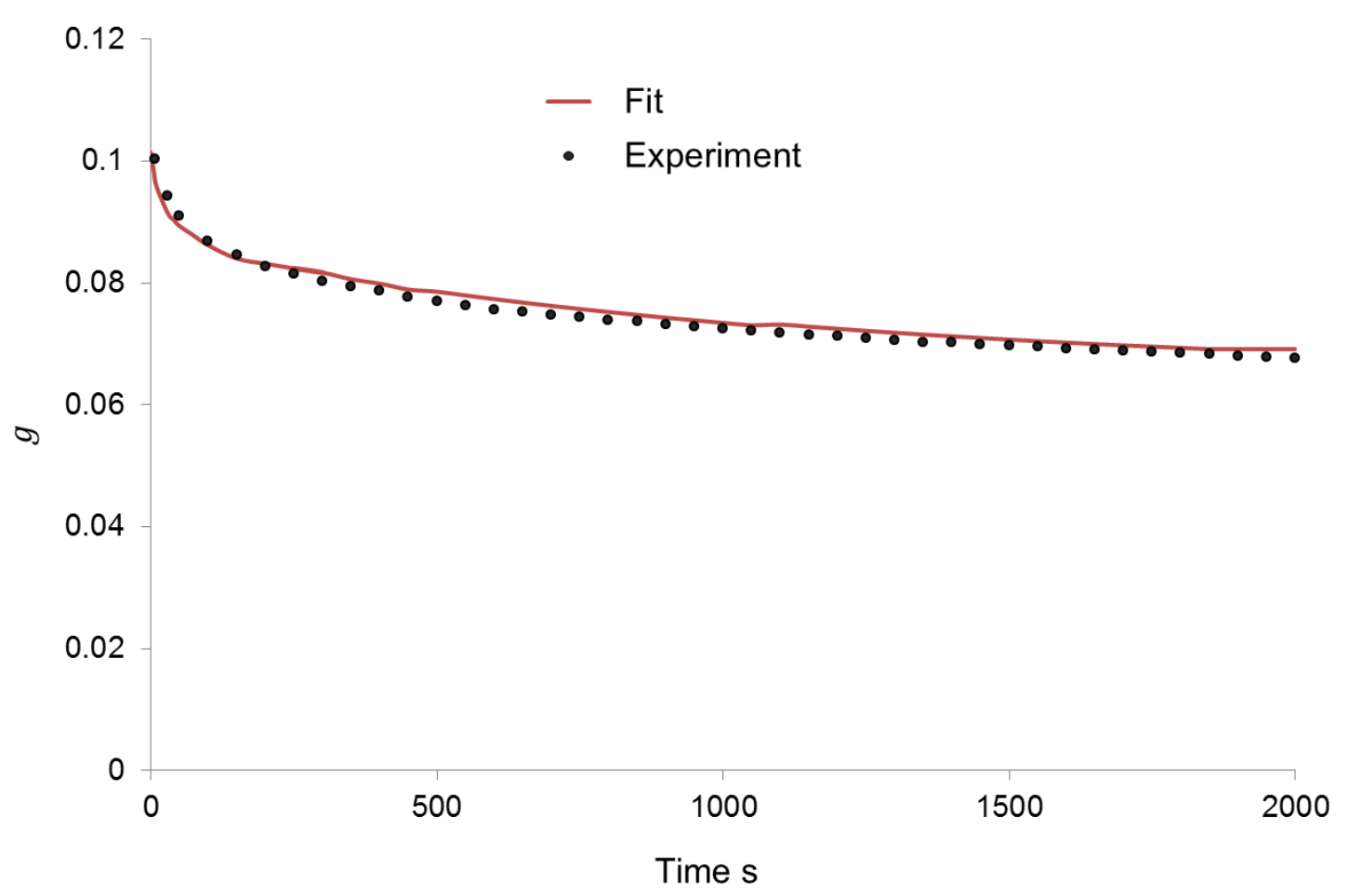

Figure 9 Normalized shear relaxation moduli, $g$, vs. time data (obtained at the strain rate of $0.1 / \mathrm{s}$ ) fitted to the GM model to find the viscoelastic parameters

Three indentations were performed on the sample, with the results of one nanoindentation test shown in Figure 10. The inversely obtained material properties were determined over the three experiments individually and then averaged. The isotropic model developed in Section 3.1 was used to predict the time dependent depth penetration for the nano-indentation using the material parameters determined from the stress relaxation test (Table 1). It can be seen from Figure 10 that there is good correspondence in the trends for the relaxation, but the loading part shows a significant discrepancy. One potential reason for this can be found in the reported orthotropy of the cornea, which is made up of fibres embedded in a matrix, with the fibres orientated within the plane of the cornea. Schematics of the fibre and load directions in the nano-indentation and relaxation tests are provided in Figure 6 and 
Journal of the Mechanical Behavior of Biomedical Materials

Figure 11, respectively. As the tensile relaxation test is performed in the fibre direction, the characterisation is perhaps not representative of a deformation during nano-indentation, which will have a large component along the fibre orientation. 

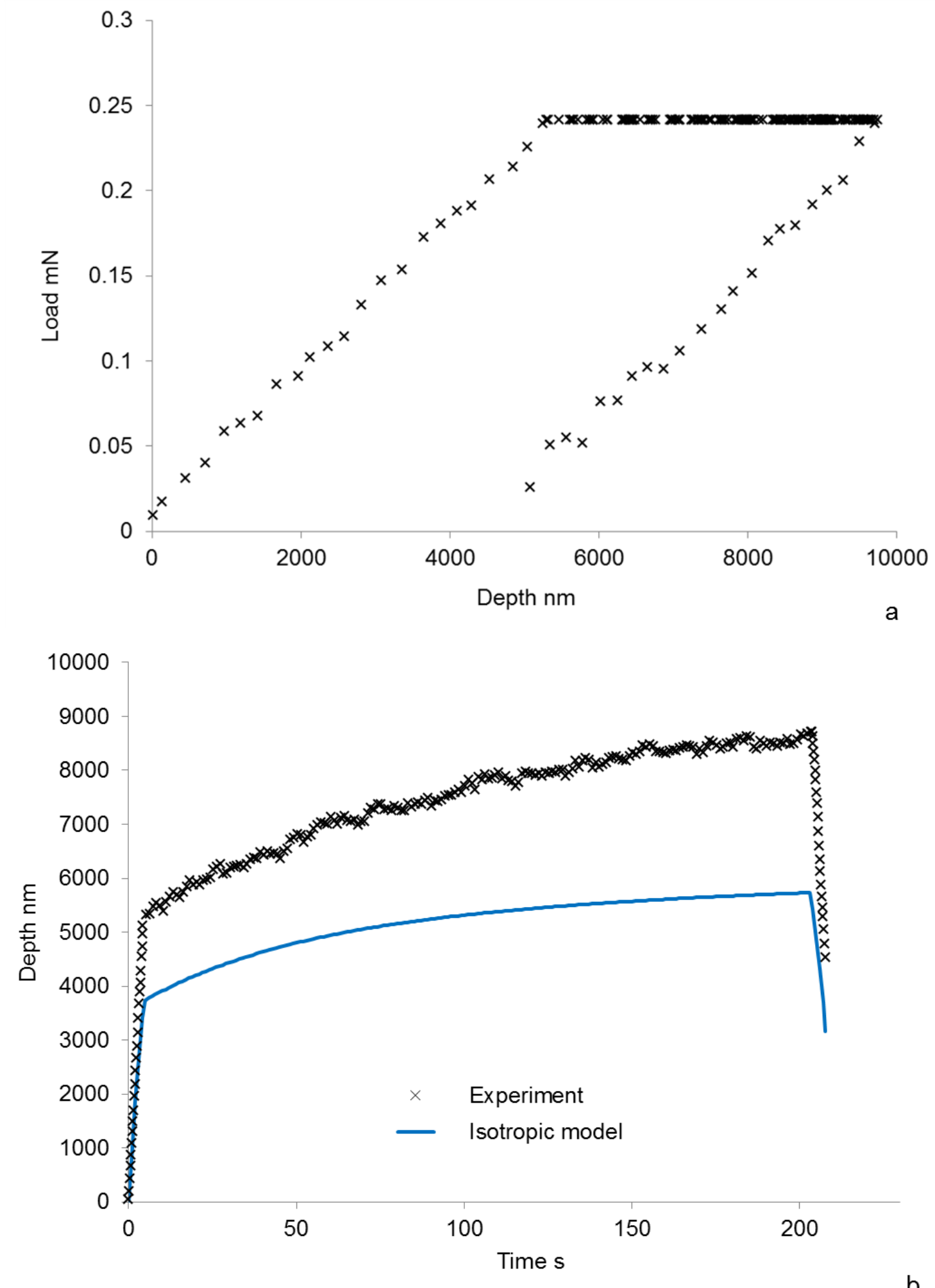

Figure 10 Nano-indentation results at the centre of the right porcine cornea, a) Load vs. depth experimental data, b) Depth vs. time experimental data in comparison with the simulation results obtained from the isotropic model 


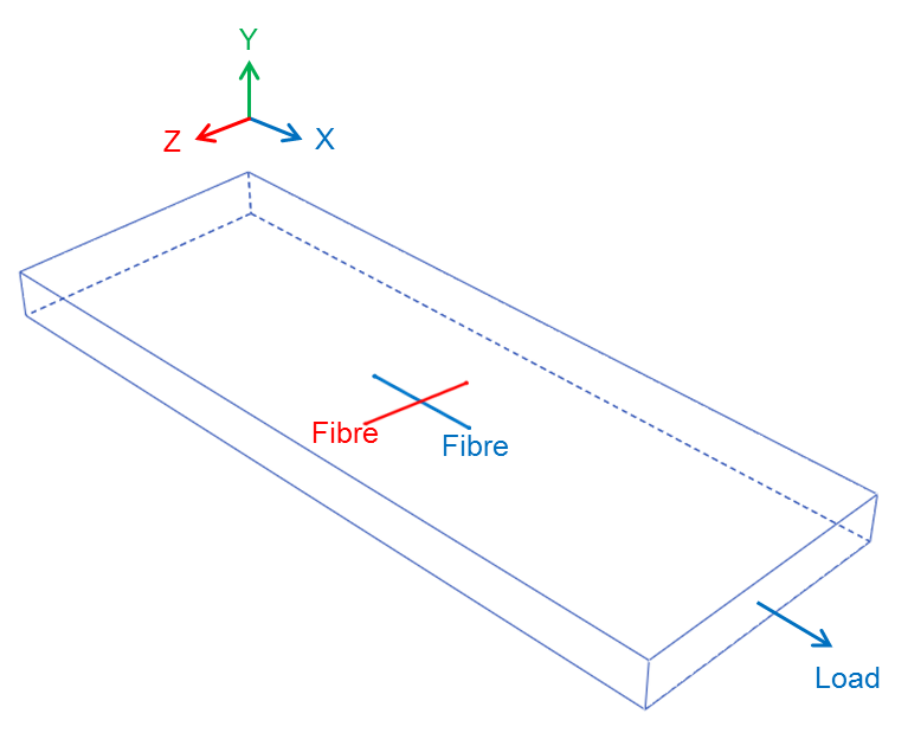

Figure 11 Alignment of corneal fibres to load direction in the stress relaxation, showing parallel loading direction to fibres

An alternative to the determination of the material properties via the stress relaxation test is to use nano-indentation combined with the inverse finite element method using a sequential application of the isotropic and orthotropic models. First, the isotropic model was used to recover the viscoelastic parameters. Later, since the viscous time-dependant parameters would be unchanged by the choice of orthotropy, a more computationally efficient inverse analysis was performed to recover orthotropic elastic parameters. The second stage was limited to the loading mode only, which allowed characterization of the two in-plane (along fibre directions) orthotropic elastic constants. The out-of-plane (along loading direction) orthotropic constant is recovered from the isotropic model. The corresponding corneal parameters were recovered from the three indentation data and the average values are provided in Table 1. The profile obtained once the inverse algorithm had converged is shown in Figure 12, demonstrating good agreement. 
Journal of the Mechanical Behavior of Biomedical Materials
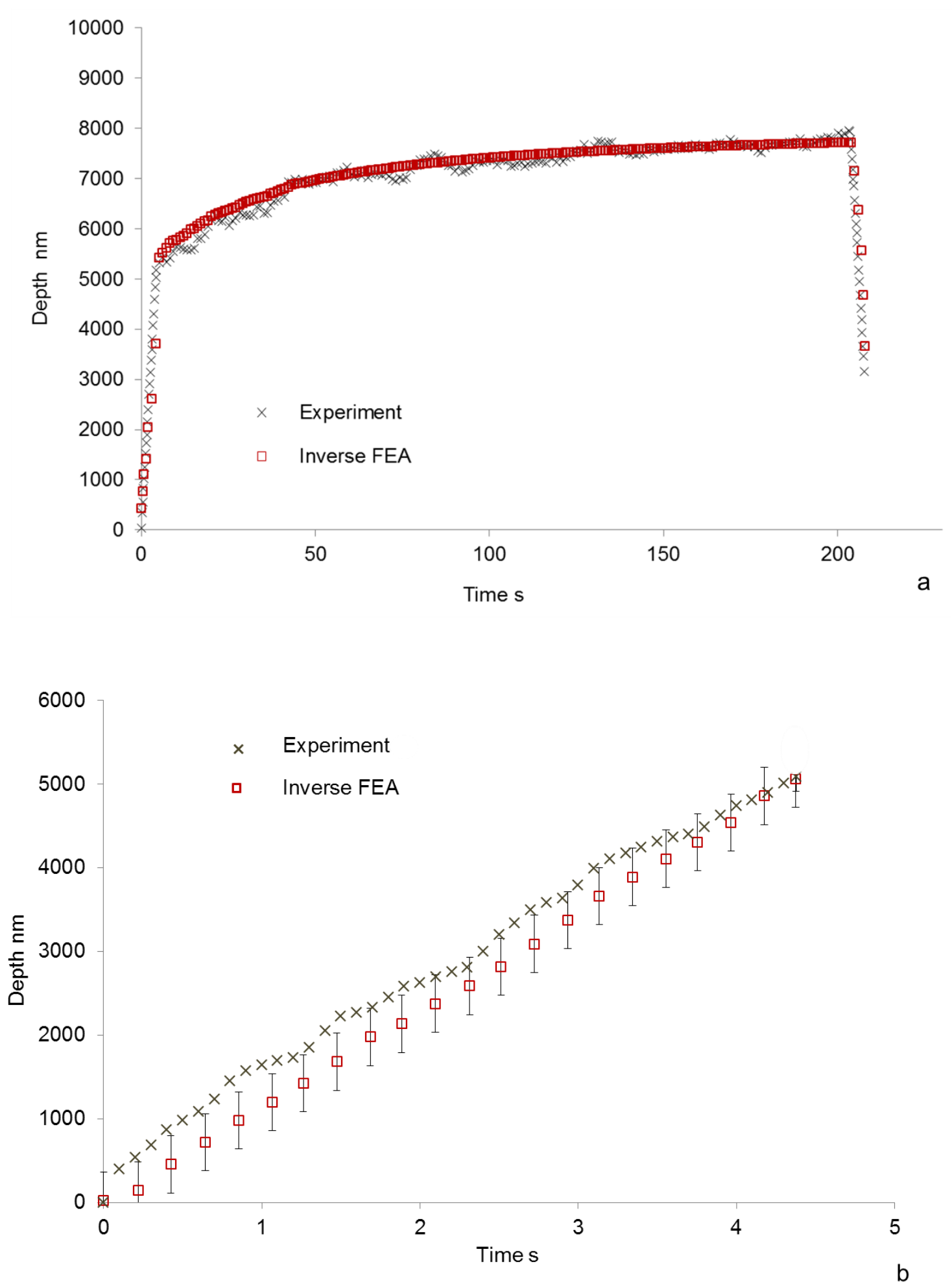

Figure 12 Converged profiles obtained from the inverse method in compare with the experiment, a) Isotropic model, b) Orthotropic model (loading-part) 
Journal of the Mechanical Behavior of Biomedical Materials

Table 1 Parameters of the corneal rheological model obtained from the stress

relaxation experiment, using curve fitting and the nano-indentation experiment $(\mathrm{NI})$

using the inverse finite element method (inverse-FEM). $E_{y}$ and long-term viscoelastic parameters are recovered from an isotropic model. $E_{x}$ and $E_{z}$ are recovered from an orthotropic model.

$$
E(M P a)
$$

Method

$$
g_{1} \quad g_{2} \quad \tau_{1}(s) \quad \tau_{2}(s)
$$

$E_{x} \quad E_{z} \quad E_{y}$

Relax. experiment

0.250

0.351

0.437

410.5

1605.3

NI inverse-FEM

$0.257 \quad 0.260$

0.153

0.332

0.454

399.1

1621.0

$\pm S T D$

$\pm 0.04 \pm 0.08$

$\pm 0.06$

$\pm 0.03$

$\pm 0.05$

$\pm 1.00$

$\pm 7.50$

Orthotropic model

Isotropic model

It can be seen from Table 1 that the fibre direction moduli, $E_{x}$ and $E_{z}$, are close to the values obtained from the relaxation test, but the normal direction modulus, $E_{y}$, is significantly smaller. This offers support for the explanation for that the discrepancies lie in the composite nature of the material, since it suggests that the normal direction is more compliant than the in-plane directions, as one might expect when fibres are orientated within the plane. These results suggest that an orthotropic linear viscoelastic model is an appropriate description of corneal behaviour, since the composite-like structure leads to direction dependent properties.

Interestingly, the values of the instantaneous elastic moduli are in the same order of magnitude with those obtained in the study of Kampmeier et al. and Ahearne et al., 
i.e. between 0.1 to 0.5MPa, (Kampmeier et al. 2000, Ahearne et al. 2007). The values for viscoelastic time constants, however, are different, suggesting that the time-dependent properties may be more susceptible to conditioning than the elastic parts.

To summarize, a flow diagram is provided in Figure 13 that indicates the proposed method of determining corneal properties.

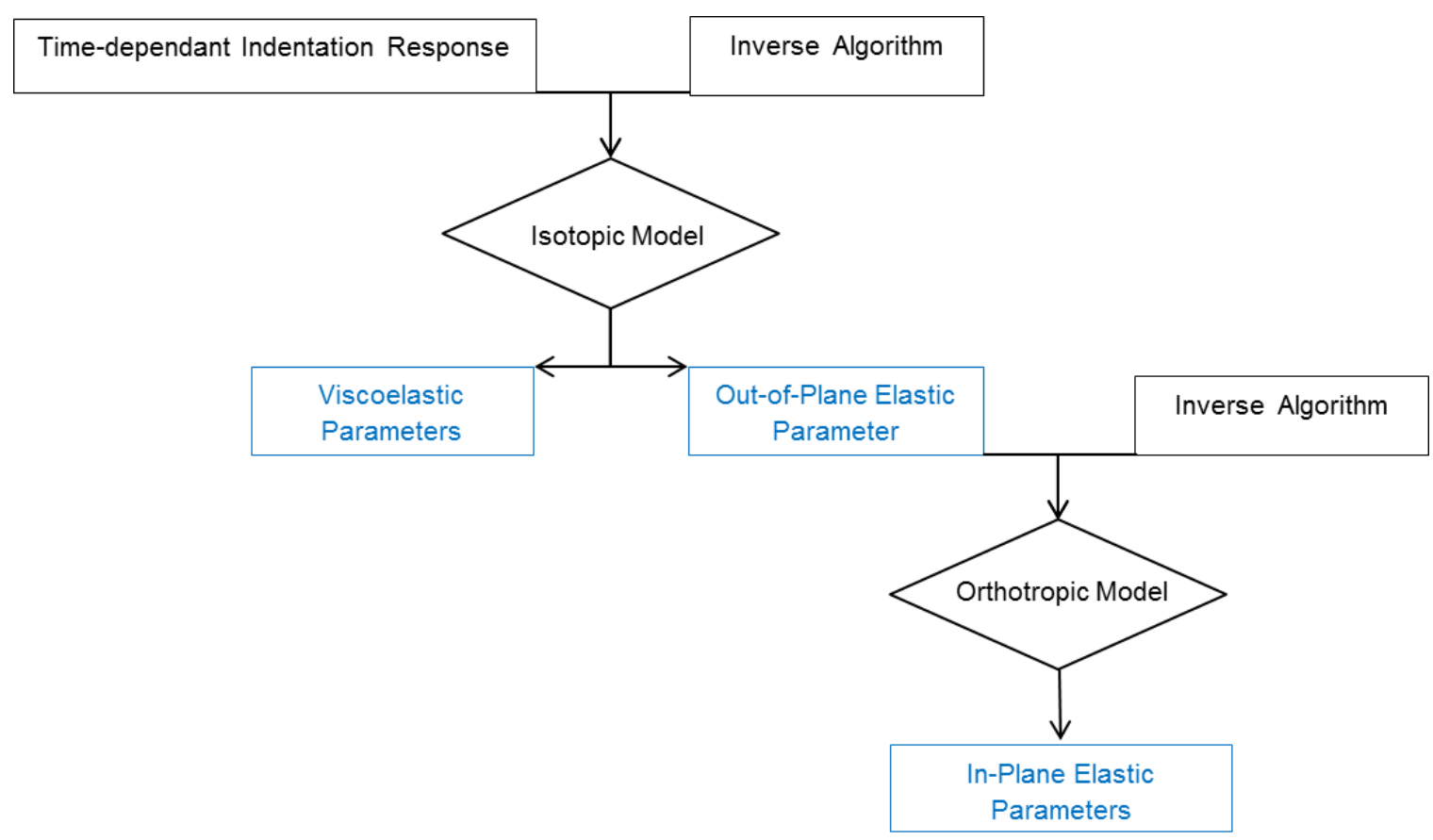

Figure $13 \mathrm{~A}$ flow diagram of the method developed in this research to determine corneal material properties

\section{Conclusion}

In this study, a method is proposed and examined to determine corneal properties invivo. The principle of corneal indentation is used in the clinical measurement of the intra-ocular pressure. Hence, our proposed method can be expanded to the in vivo measurement of the corneal material properties. This method is based on a 
combination of a nano-indentation technique and inverse finite element analysis. The time-dependant corneal response, as an input to the inverse algorithm, was obtained from the nano indentation experiment and the parameters of the corneal model were recovered using the inverse finite element method. To investigate the performance of the inverse method, the recovered parameters were checked against the fitted parameters from the stress relaxation experiment.

The use of a GM rheological model for the cornea enables the instantaneous elastic behaviour be separated from the long-term viscoelastic behaviour. It was shown that the instantaneous elastic behaviour is dependent on the type of experiment and the corneal composite microstructure. Hence, an orthotropic rheological model is required to fully characterize the time-dependant behaviour of the cornea. Further investigations on the application of the inverse method combined with the nanoindentation technique for obtaining an inhomogeneity map of the cornea can be the prospect of future studies.

\section{Acknowledgments}

The authors wish to thank Mr T. Nguyen and Mr A. Alghamdi for their useful discussions and Loughborough University for funding this project. 


\section{References}

Ahearne, M., Yang, Y., Then, K.Y. \& Liu, K.K. 2007, "An indentation technique to characterize the mechanical and viscoelastic properties of human and porcine corneas", Annals of Biomedical Engineering. 35, 9, 1608-1616.

Boyce, B.L., Jones, R.E., Nguyen, T.D. \& Grazier, J.M. 2007, "Stress-controlled viscoelastic tensile response of bovine cornea", Journal of Biomechanics. 40, 11, 2367-2376.

Boyce, B.L., Grazier, J.M., Jones, R.E. \& Nguyen, T.D. 2008, "Full-field deformation of bovine cornea under constrained inflation conditions", Biomaterials. 29, 28, 3896-3904.

Cheng, L., Xia, X., Scriven, L. \& Gerberich, W. 2005, "Spherical-tip indentation of viscoelastic material", Mechanics of Materials. 37, 1, 213-226.

Crabb, R.A.B., Chau, E.P., Evans, M.C., Barocas, V.H. \& Hubel, A. 2006, "Biomechanical and microstructural characteristics of a collagen film-based corneal stroma equivalent", Tissue engineering. 12, 6, 1565-1575.

Dunn, M.G. \& Silver, F.H. 1983, "Viscoelastic behavior of human connective tissues: relative contribution of viscous and elastic components", Connective tissue research, 12, 1, 59-70.

Edmund, C. 1988, "Corneal elasticity and ocular rigidity in normal and keratoconic eyes", Acta Ophthalmologica. 66, 2, 134-140.

Elsheikh, A., Wang, D. \& Pye, D. 2007, "Determination of the modulus of elasticity of the human cornea", Journal of refractive surgery (Thorofare, N.J.: 1995). 23, 8, 808-818.

Friswell, M.I. \& Mottershead, J.E. 1995, Finite element model updating in structural dynamics, second ed. Springer

Fung, Y. 1993, Biomechanics: mechanical properties of living tissues, second ed. Springer.

Glass, D.H., Roberts, C.J., Litsky, A.S. \& Weber, P.A. 2008, "A Viscoelastic Biomechanical Model of the Cornea Describing the Effect of Viscosity and Elasticity on Hysteresis", Invest.Ophthalmol.Vis.Sci.. 49, 9, 3919-3926.

Grédiac, M., Pierron, F., Avril, S. \& Toussaint, E. 2006, "The Virtual Fields Method for Extracting Constitutive Parameters From Full-Field Measurements: a Review", Strain. 42, 4, 233-253.

Hayashi, K., Yoshida, M. \& Hayashi, H. 2009, "Postoperative corneal shape changes: microincision versus small-incision coaxial cataract surgery", Journal of Cataract \& Refractive Surger. 35, 2, 233-239. 
Hoeltzel, D.A., Altman, P., Buzard, K. \& Choe, K. 1992, "Strip extensiometry for comparison of the mechanical response of bovine, rabbit, and human corneas", Journal of Biomechanical Engineering. 114, 2, 202-215.

Itskov, M. \& Aksel, N. 2002, "Elastic constants and their admissible values for incompressible and slightly compressible anisotropic materials", Acta Mechanica. 157, 1,. 81-96.

Kaliske, M. 2000, "A formulation of elasticity and viscoelasticity for fibre reinforced material at small and finite strains", Computer Methods in Applied Mechanics and Engineering. 185, 2, 225-243.

Kampmeier, J., Radt, B., Birngruber, R. \& Brinkmann, R. 2000, "Thermal and biomechanical parameters of porcine cornea", Cornea. 19, 3, 355.

Levenberg, K. 1944, "A method for the solution of certain nonlinear problems in least squares", Quart.Appl.Math. 2, 2, 164-168.

Liu, X., Elmahdy, A., Wildman, R., Ashcroft, I. \& Ruiz, P. 2011, "Experimental investigation and material modelling of fresh and UV aged Japanese lacquer ", Progress in Organic Coatings. 70, 4, 160-169.

Nguyen, T. \& Boyce, B. 2011, "An inverse finite element method for determining the anisotropic properties of the cornea", Biomechanics and Modeling in Mechanobiology. 10, 3, 323-337.

Nguyen, T.D., Jones, R.E. \& Boyce, B.L. 2008, "A Nonlinear Anisotropic Viscoelastic Model for the Tensile Behavior of the Corneal Stroma", Journal of Biomechanical Engineering. 130, 4, 041020.

Nickerson, C.S. 2006, "Engineering the mechanical properties of ocular tissues", Journal of Biomechanical Engineering. 98, 2, 330-343.

Oyen, M. 2006, "Analytical techniques for indentation of viscoelastic materials", Philosophical magazine, 86, 33, 5625-5642.

Pinsky, P.M., van der Heide, D. \& Chernyak, D. 2005, "Computational modeling of mechanical anisotropy in the cornea and sclera", Journal of Cataract \& Refractive Surgery, 31, 1,. 136-145.

Yang, S., Zhang, Y.W. \& Zeng, K. 2004, "Analysis of nanoindentation creep for polymeric materials", Journal of Applied Physics, 95, 7, 3655-3666.

Zadok, D., Tran, D.B., Twa, M., Carpenter, M. \& Schanzlin, D.J. 1999, "Pneumotonometry versus Goldmann tonometry after laser in situ keratomileusis for myopia", Journal of Cataract \& Refractive Surgery, 25, 10, 1344-1348. 
Zeng, Y., Yang, J., Huang, K., Lee, Z. \& Lee, X. 2001, "A comparison of biomechanical properties between human and porcine cornea", Journal of Biomechanics. 34, 4, 533-537.

Zhou, Y., Wu, Z., Ge, J., Wan, P., Li, N., Xiang, P., Gao, Q. \& Wang, Z. 2011, "Development and characterization of acellular porcine corneal matrix using sodium dodecylsulfate", Cornea. 30, 1, 73-82. 